\title{
Local Design Principles at Hippocampal Synapses Revealed by an Energy-Information Trade-Off
}

\author{
DGaurang Mahajan and $\mathbb{D}^{-S u h i t a ~ N a d k a r n i}$
}

https://doi.org/10.1523/ENEURO.0521-19.2020

Indian Institute of Science Education and Research, Pune 411 008, India

\begin{abstract}
Synapses across different brain regions display distinct structure-function relationships. We investigated the interplay of fundamental design constraints that shape the transmission properties of the excitatory CA3-CA1 pyramidal cell connection, a prototypic synapse for studying the mechanisms of learning in the mammalian hippocampus. This small synapse is characterized by probabilistic release of transmitter, which is markedly facilitated in response to naturally occurring trains of action potentials. Based on a physiologically motivated computational model of the rat CA3 presynaptic terminal, we show how unreliability and short-term dynamics of vesicular release work together to regulate the trade-off of information transfer versus energy use. We propose that individual CA3-CA1 synapses are designed to operate near the maximum possible capacity of information transmission in an efficient manner. Experimental measurements reveal a wide range of vesicular release probabilities at hippocampal synapses, which may be a necessary consequence of long-term plasticity and homeostatic mechanisms that manifest as presynaptic modifications of the release probability. We show that the timescales and magnitude of short-term plasticity (STP) render synaptic information transfer nearly independent of differences in release probability. Thus, individual synapses transmit optimally while maintaining a heterogeneous distribution of presynaptic strengths indicative of synaptically-encoded memory representations. Our results support the view that organizing principles that are evident on higher scales of neural organization percolate down to the design of an individual synapse.
\end{abstract}

Key words: efficient signaling; hippocampal representation; information theory; short-term plasticity; synaptic facilitation; synaptic failures

\section{Significance Statement}

Synapses across the brain widely vary in morphology and dynamics, suggesting diversity in underlying design principles. The Schaffer collateral-CA1 synapse is a crucial component of the hippocampal circuit associated with learning. We used information transmission and energy utilization, fundamental constraints that govern neural organization, to gain insights into the form-function relationship at this synapse which is characterized by unreliable neurotransmitter release. We show that short-lasting activity-dependent enhancement, a distinguishing attribute of this synapse, ensures that information carried by transmitter release is maximized in an energetically cost-effective manner. Remarkably, we find that synapse-specific quirks ensure information rate is independent of the release probability; thus, even as ongoing long-term memory storage continues to feed heterogeneity in presynaptic strengths, individual synapses maintain robust information transmission. Our analysis reveals the unique design compromises implicit in the distinctive features of this synapse, sharing design principles with higher levels of brain organization.

Received December 11, 2019; accepted March 17, 2020; First published August 26, 2020.

The authors declare no competing financial interests.
Author contributions: G.M. and S.N. designed research; G.M. performed research; G.M. and S.N. analyzed data; G.M. and S.N. wrote the paper. 


\section{Introduction}

Chemical synaptic transmission accounts for a significant proportion of metabolic costs during normal neural activity in the mammalian brain (Attwell and Laughlin, 2001). Understanding the role of competing demands imposed by energy consumption and information processing in shaping nervous systems has been an enduring question in neuroscience research (Laughlin, 2001; Balasubramanian et al., 2001; Laughlin and Sejnowski, 2003; Hasenstaub et al., 2010); one may then ask if the notion of energetic efficiency trickles down to the level of individual synapses. In this context, failures of transmitter release, while unsuccessful in relaying presynaptic action potentials, may help conserve synaptic resources by lowering average release rates. Indeed, probabilistic release is a characterizing feature found across a number of synapses (Borst, 2010), and a fundamental source of stochasticity in neural dynamics (Deco et al., 2009). Previous studies have suggested that synaptic failures support both efficient neural coding (Levy and Baxter, 2002) and communication between neurons (Harris et al., 2012), but these studies did not include the effect of use-dependent short-term plasticity (STP) that typically accompanies probabilistic release and which can significantly modulate the time course of synaptic responses to natural activity patterns (Tsodyks and Markram, 1997; Matveev and Wang, 2000; Zucker and Regehr, 2002).

Excitatory Schaffer collateral-CA1 pyramidal cell connections, a crucial component of the hippocampal circuitry engaged during spatial navigation and implicated in experience-dependent learning (Gruart et al., 2006; Basu and Siegelbaum, 2015; Choi et al., 2018), provide a distinctive example of low release probability synapses (Allen and Stevens, 1994). Individual synapses show strong enhancement of release probabilities in response to natural spike trains (Dobrunz and Stevens, 1999), in contrast to their low transmission rates for single spikes; this short-term facilitation (STF) is observed over timescales of milliseconds to seconds. It was previously proposed that the CA3-CA1 pathway is optimized for conveying information on spike times in short bursts occurring at physiologically relevant frequencies (Rotman et al., 2011). However, the concomitant energy costs associated with vesicular release and recycling supporting this form of transmission are not known.

Here, we use a computational model to investigate the relevance of energetic constraints to the design and function of single hippocampal synapses that are characterized

This work was supported by the Science \& Engineering Research Board, India, Grant PDF/2017/001803 (to G.M.) and the Wellcome Trust/DBT India Alliance, Grant IA/I/12/1/500529 (to S.N.).

Acknowledgements: We thank IISER, Pune for supporting this study. G.M. acknowledges helpful discussions on an earlier version of this work at the OIST Computational Neuroscience Course 2018.

Correspondence should be addressed to Suhita Nadkarni at suhita@ iiserpune.ac.in.

https://doi.org/10.1523/ENEURO.0521-19.2020

Copyright @ 2020 Mahajan and Nadkarni

This is an open-access article distributed under the terms of the Creative Commons Attribution 4.0 International license, which permits unrestricted use, distribution and reproduction in any medium provided that the original work is properly attributed. by low initial release probabilities but marked activity-dependent STP. Our study addressing individual synapses is thus distinct from earlier work based on synaptic population readouts (Klyachko and Stevens, 2006; Rotman et al., 2011). Other previous studies of information transmission at cortical synapses considered short-time dynamics arising from vesicle depletion alone (Manwani and Koch, 2001; Goldman, 2004; Rosenbaum et al., 2012; Salmasi et al., 2019a), or made simplifying model assumptions about presynaptic organization (e.g., availability of at most one vesicle per release site; Harris et al., 2012; Zhang and Peskin, 2015; Salmasi et al., 2019b), limiting their physiological relevance for describing facilitating hippocampal synapses. Another distinguishing feature of our study is that we do not ascribe a notion of information to "a" spike as is often done (Goldman, 2004; Rotman et al., 2011; Harris et al., 2012), as it is unclear whether every presynaptic spike can be assigned equivalent meaning at the CA3-CA1 synapse. In the hippocampus, neural information may be encoded by brief increases in firing rate rather than the precise timing of individual spikes. A relevant example is provided by the selective activation of specific subsets of CA3 pyramidal cells whenever the animal enters their preferred spatial location (O'Keefe and Dostrovsky, 1971); such brief increases in firing frequency punctuating a low-activity background are regarded as discrete units of information (Lisman, 1997), which may be further modulated by additional task-relevant variables (Fenton and Muller, 1998). Thus, instead of a spike-centric approach assessing how reliably presynaptic spike times are conveyed to the target cell, we make the conceptual distinction that information is more correctly an attribute of the underlying physiologically identifiable temporal "signal" encoded in the irregular firing activity of the presynaptic neuron.

Vesicular release properties are seen to vary widely across synapses, being tuned to the functional demands of the circuits in which they are embedded (Thomson, 2000; Dittman et al., 2000; Abbott and Regehr, 2004). Thus, addressing design principles on a generic level is hindered by the diversity of synapse types found in the nervous system and the neural activity patterns that they process. How a small hippocampal synapse defined by low release probability and a limited pool of available vesicles, equipped with STP, regulates the local balance between reliability and economy of signaling in a physiological setting has not been addressed thus far. Our model includes relevant biological details and characterizes the role of activity-dependent, short-term release dynamics in modulating the transmission of rate-coded behaviorally relevant presynaptic signals at single synapses, providing an apt description of the Schaffer collateral pathway that is characterized by a preponderance of monosynaptic connections between CA3/CA1 cell pairs (Debanne et al., 1995). CA3 synaptic populations display considerable heterogeneity in their transmitter release properties (Allen and Stevens, 1994; Dobrunz and Stevens, 1997; Holderith et al., 2012), and we particularly sought to address how these differences among synapses impact their ability to relay information-carrying spike trains. We show that the vesicle code operates within a 


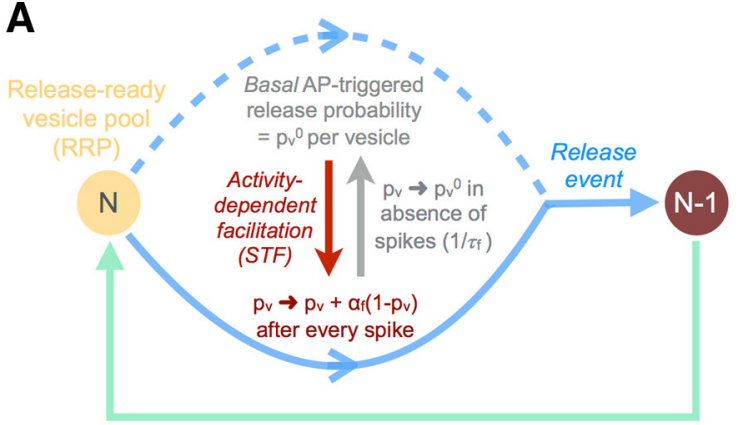

Replacement rate per vesicle $\sim 1 / \tau_{\mathrm{r}}$
B

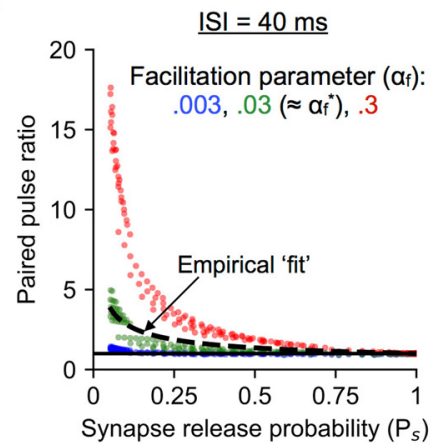

$\underline{|S|=10-1000 \mathrm{~ms}}$

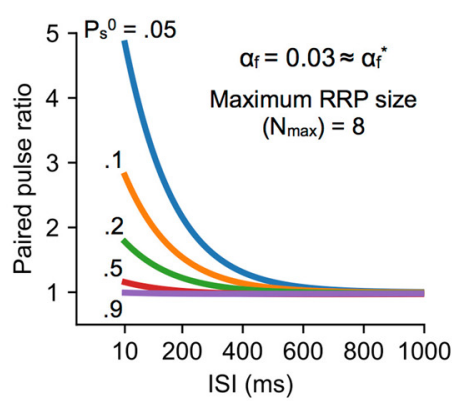

C

Presynaptic spiking at $30 \mathrm{~Hz}$ Maximum RRP size $\left(\mathrm{N}_{\max }\right)=8$

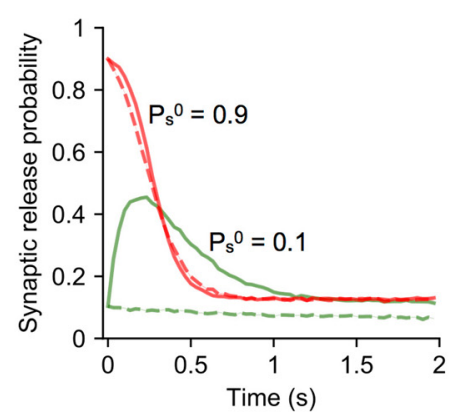

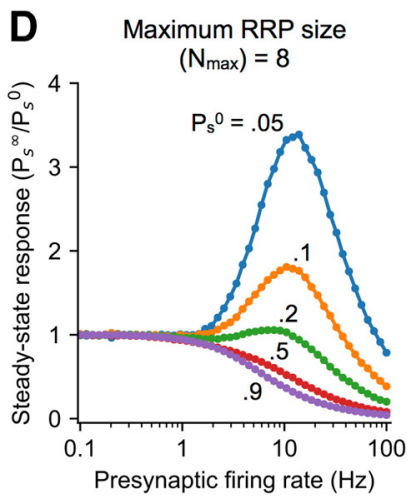

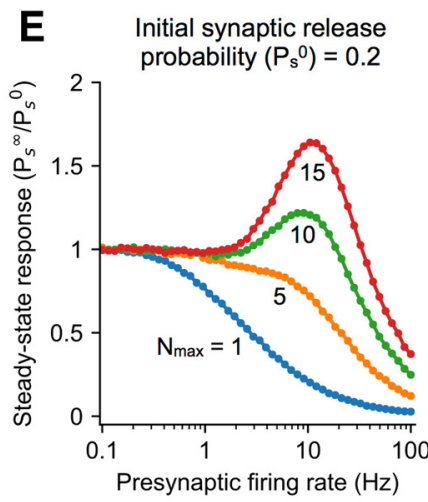

Figure 1. Modeling short-term plasticity (STP) at stochastic hippocampal synapses. A, Outline of the reduced model of presynaptic STP used in the study, which includes activity-dependent facilitation of neurotransmitter release and depression because of slower recovery of released vesicles. Facilitation strength is tuned by the dimensionless gain parameter $\alpha_{f}$. B, left, Distribution of PPRs over a realistic range of RRP sizes (1-15) and basal per-vesicle release probabilities ( $p_{v}^{0}$ ranging from $10^{-4}$ to 1 ) in the STP model $(I S I=40 \mathrm{~ms})$. Different magnitudes of facilitation $\left(\alpha_{f}\right)$ are represented by different colors. The dashed curve captures the empirical distribution of PPR values (from Dobrunz and Stevens, 1997), and the solid black line corresponds to PPR $=1$. Right, Dependence of PPR on the interspike interval (ISI) for representative facilitating synapses with the same maximum RRP size and different basal transmitter release probabilities. $\boldsymbol{C}$, Example of STP dynamics at realistic synapses with low $\left(P_{s}^{0}=0.1\right)$ and high $\left(P_{s}^{0}=0.9\right)$ initial release probability during the response to sustained presynaptic spiking at $30 \mathrm{~Hz}$. Solid and dashed curves correspond to synapses with facilitation and lacking facilitation (constant $p_{v}$ ), respectively. Results shown as mean $\pm \mathrm{SEM}$ over $10^{4}$ independent trials; $N_{\text {max }}=8$. $\boldsymbol{D}$, Regimes of facilitation and depression illustrated by the frequency dependence of normalized asymptotic/steady-state response for synapses with different basal failure rates (different colors). All synapses have the same maximum RRP size of 8 . Results shown as mean \pm SEM over $10^{4}$ trials per parameter combination. $\boldsymbol{E}$, Dependence of synaptic filtering on the number of available vesicles illustrated by the frequency-response curves for synapses with the same initial release probability $\left(P_{s}^{0}=0.2\right)$ and varying maximum RRP size (different colors represent different $N_{\max }$ ). Results shown as mean \pm SEM over $10^{4}$ trials per parameter combination.

range that maintains low energy costs while maximizing information dynamically via STP for the entire heterogeneous population of intrinsic release probabilities seen at these synapses.

\section{Materials and Methods}

Experimental studies have provided valuable information about the ultrastructural organization and distribution of dynamic properties at rat CA3-CA1 presynaptic terminals (Dobrunz and Stevens, 1997; Schikorski and Stevens, 1997). Individual CA3 presynaptic boutons typically have a single active zone (Schikorski and Stevens, 1997), where glutamate release occurs in a probabilistic manner (Allen and Stevens, 1994) and shows a complex mix of use-dependent depression because of refractoriness in vesicle recovery and rapid calcium-mediated facilitation of the release machinery (Stevens and Wang, 1995; Dittman et al., 2000). We adopted a mathematical description of this synapse which captures key attributes of its short-time release dynamics (Fig. $1 A$ ), and quantified through numerical simulations its responses to irregular spike trains mimicking naturally occurring presynaptic cell activity. The model details and setup for our analysis are briefly described below.

\section{Estimating synaptic information rates}

Experimental recordings from rodent hippocampus suggest that individual pyramidal cells in area CA3 show location-specific (place cell) firing during free exploration (Fenton and Muller, 1998; Mizuseki et al., 2012). Further, it has been argued that variability seen in these brief increases in firing during individual passes through the preferred location may encode additional attributes, such as aspects of the animal's trajectory (Allen et al., 2012; Grieves et al., 2016), its motion relative to goal direction (Aoki et al., 2019), variable attentional state of the animal (Olypher et al., 2002; Fenton et al., 2010), modulation by 
contextual cues (Shapiro et al., 1997; Leutgeb et al., 2005) (besides simply the variable duration, or equivalently, the running speed, at each pass), etc. These observations motivate our model wherein we consider synaptic processing of integrated spatial and contextual signals represented by the occurrences and variable size (number or frequency of spikes) of place field (PF) discharges of the CA3 neuron. We thus define an effective "input" signal, $\phi(t)$, underlying the variable firing activity of the presynaptic cell which is assumed to be zero everywhere except whenever the trajectory crosses the cell's preferred location. Every pass through the PF is associated with a non-zero value of $\phi, \phi_{i}$, which in our model is sampled from a uniform random distribution on the interval $\left[\phi_{\min }, \phi_{\text {max }}\right]$. This range is adjusted to be compatible with the statistics of experimentally recorded hippocampal spike train data (described below).

Individual PF passes are assumed to be uncorrelated in time and to occur sparsely, at a mean rate of $r_{s} \mathrm{~s}^{-1}$ (hereafter, we use the term "input rate" to refer to the mean rate of PF passes). In order to estimate the information rate carried by $\phi(t)$, time is uniformly divided into sufficiently short steps of $\Delta t\left(\ll 1 / r_{s}\right)$, which is taken to be the (fixed) duration of every pass; every time step is thus treated as an independent realization of the probability distribution of $\phi, \mathrm{P}(\phi)$, that is determined by the value of $r_{s}$. The time-averaged, discretized, entropy rate of the input signal is quantified in the usual manner using Shannon's measure (Cover and Thomas, 2012):

$$
\mathcal{R}_{s}=-(1 / \Delta t) \sum_{\phi} P(\phi) \log _{2} P(\phi)(\text { bits } / \mathrm{s})
$$

where the sum runs over all values that $\phi$ can assume, with $\mathrm{P}(\phi)$ being approximated by a discrete distribution over $\mathrm{n}_{\phi}$ possible states (set to 20 in our analysis). The value of $\phi$ during every PF pass determines the corresponding burst size; the number of spikes comprising every burst is thus given by a second, conditional, Poisson distribution with mean $\lambda(\phi)$ (the exact form of which depends on the specific interpretation of the $\phi$ variable; see "Model implementation" below), and these spikes are assumed to occur at random times within the corresponding pass of duration $\Delta \mathrm{t}$. To be consistent with experimental data, a small amount of "noise" is also added to the system, modeled as a constant background presynaptic spiking rate of $r_{n} s^{-1}$ (this spiking is uncorrelated with the spatial context and could arise, say, from synaptic or channel noise).

The synaptic response to presynaptic spike patterns consists of a sequence of evoked transmitter release events, and we quantify how well this discrete temporal sequence conveys the temporal modulation of the signal $\phi$ underlying the irregular firing behavior of the presynaptic neuron. By analogy with the input, we discretize the CA3 synaptic output by binning the releases occurring within every time step $\Delta t$. Thus, every burst evokes a variable amount of transmitter, proportional to the number of release events, $n_{r}$, and the vesicular response profile is given by a sequence of $n_{r}$ values (one number per $\Delta$ t step, and that are assumed to produce graded postsynaptic responses via temporal summation of EPSPs or NMDA receptor-gated $\mathrm{Ca}^{2+}$ transients). Under the assumption of low noise level, synaptic transmission is characterized by the stationary joint probability distribution $\mathrm{P}\left(\phi, n_{r}\right) \equiv \mathrm{P}(\phi)$ $\mathrm{P}\left(n_{r} \mid \phi\right)$ which is (implicitly) sampled at every time step in our simulations. The conditional distribution $\mathrm{P}\left(n_{r} \mid \phi\right)$ is governed by the form of the synaptic dynamics used in the model and encapsulates the effects of STP. Synaptic responses to successive bursts can be considered to be uncorrelated, which is a valid approximation when the typical interval between PF passes is longer than the slowest timescale in the model of synaptic dynamics (this is set by the recovery rate of the release-ready vesicle pool; see below). We characterize the fidelity of information coding at an individual synapse in terms of a discretized version of the average mutual information rate $\left(\mathcal{R}_{r s}\right)$, a standard nonparametric measure of statistical relatedness of two variables, which can be expressed as a difference between the total entropy of the synaptic response and the noise entropy (Cover and Thomas, 2012):

$$
\begin{gathered}
\mathcal{R}_{r s}=-(1 / \Delta t) \times \\
{\left[\sum_{n_{r}} P\left(n_{r}\right) \log _{2} P\left(n_{r}\right)-\sum_{\phi} \sum_{n_{r}} P(\phi) P\left(n_{r} \mid \phi\right) \log _{2} P\left(n_{r} \mid \phi\right)\right]} \\
(\text { bits } / \mathrm{s}) .
\end{gathered}
$$

This rate is numerically estimated from the pooled data from simulations run for sufficiently long duration.

\section{Estimating efficiency of the vesicle code}

Following previous studies (Goldman, 2004; Harris et al., 2012; James et al., 2019), local energetic efficiency of the synaptic code is quantified in terms of the average number of releases per bit transmitted per synapse (i.e., the specific cost of information), and we use the measure $E\left(s^{-1}\right)=\mathcal{R}_{\text {ves }} /\left(\mathcal{R}_{r s} / \mathcal{R}_{s}\right)$, where $\mathcal{R}_{\text {ves }}$ denotes the mean rate of fusion events at the synapse (averaged over every simulation run). $\mathcal{R}_{\text {ves }}$ accounts for the use of synaptic resources during signal transmission and also provides a proxy for the net energy expenditure associated with transmitter release and recycling (Harris et al., 2012). The specific or unit cost of information as defined above is inversely related to the notion of efficiency, i.e., higher efficiency synapses are expected to require fewer vesicles to carry information at a given rate.

\section{Modeling release dynamics at probabilistic synapses}

Every synaptic release site is characterized by its basal spike-evoked transmission probability, $P_{s}^{0}$, and maximum size of the docked pool of release-ready vesicles [readily-releasable pool (RRP)], $N_{\max }$. The synaptic release probability $\left(P_{s}\right)$ is distinct from the fusion probability per docked vesicle $\left(p_{v}\right)$, and under the assumption that docked vesicles can fuse independently of each other (Dobrunz and Stevens, 1997), the two are related by $P_{s}=1-\left(1-p_{v}\right)^{N_{\text {RRP }},} N_{\text {RRP }}$ being the instantaneous RRP size at the release site.

We model the synchronous component of vesicular release evoked by presynaptic spikes (Dobrunz and Stevens, 1997) and assume that every spike can trigger 
release of at most one vesicle per synapse. This assumption of uniquantal release at glutamatergic CA3-CA1 synapses is compatible with experimental findings (Stevens and Wang, 1995) suggesting a refractory phase associated with fusion of a vesicle, which may inhibit subsequent release events in a short time period $(\sim 10 \mathrm{~ms})$ following the initial spike during which the local calcium concentration at the release site is high. Following its release, every vesicle is assumed to be recovered independently, and this replenishment is also modeled as a stochastic process with mean recovery timescale of $\tau_{r}$ per vesicle. During bouts of intense spiking activity, rapid depletion of the docked vesicle pool can occur, mediating a form of transient depression whose strength and duration are controlled by $\tau_{r}$ together with the resting pool size at the synapse. We set $\tau_{r}$ to $2 \mathrm{~s}$, consistent with experimentally measured refilling rates at rat hippocampal synapses (Klyachko and Stevens, 2006).

Transient depression because of slow recycling of released vesicles is complemented by activity-dependent changes in vesicle fusion at the release site. We adopt a reduced kinetic model (Fig. $1 A$ ) to describe this STF regulated by spike-driven calcium dynamics at the active zone, which follows from a number of previous studies of presynaptic plasticity (Tsodyks and Markram, 1997; Hennig, 2013). The spike-triggered per-vesicle release probability, $p_{v}$, is treated as a dynamical variable whose dependence on the presynaptic spiking history is governed by the following equation:

$$
\frac{d p_{v}}{d t}=\frac{\left(p_{v}^{0}-p_{v}\right)}{\tau_{f}}+\alpha_{f}\left(1-p_{v}\right) \sum_{i} \delta\left(t-t_{i}\right),
$$

with the sum running over the set of all spike times. Thus, the arrival of every presynaptic spike increments the value of $p_{v}$ by an amount proportional to the synaptic gain parameter $\alpha_{f}$, and the factor $\left(1-p_{v}\right)$ ensures that $p_{v}$, being a probability, does not exceed 1 . The first term on the RHS of Equation 1 describes the exponential relaxation of $p_{v}$ to its baseline value $p_{v}^{0}$ in the absence of spiking activity. Interspike intervals shorter than the facilitation time constant, $\tau_{f}$, are expected to induce strong enhancement of vesicle release. Following previous studies, we model a rapid form of STF with $\tau_{f}=150 \mathrm{~ms}$ (Klyachko and Stevens, 2006; Cai et al., 2007). Longer-lasting forms of presynaptic plasticity such as augmentation are not considered here; these components are normally induced in experimental settings with sustained high-frequency synaptic stimulation (Zucker and Regehr, 2002), and unlikely to be of significance during the sparse, sporadic spiking activity observed in the physiological conditions modeled here.

The form of STF given by Equation 3 implies a general trend of decreasing facilitation with increase in the basal synaptic release efficacy, which is supported by experimental recordings of individual hippocampal synapses (Dobrunz and Stevens, 1997; Murthy et al., 1997). In order to select a physiologically relevant value for the gain parameter $\alpha_{f}$, we refer to previous experimental data on pairedpulse stimulation at rat CA3-CA1 synapses [Dobrunz and Stevens, 1997; sex of animal(s) not mentioned in source publication]. This synaptic population displayed a broad range of baseline $P_{s}$ values $(\sim 0.05-1)$, and the release probabilities recorded in response to two spikes separated by a short interval $(40 \mathrm{~ms})$ yielded a distribution of paired-pulse facilitation ratios (PPRs) whose dependence on the initial synaptic release probability $\left(P_{s}\right)$ was well-fit by the relation $\mathrm{PPR}=\left(1-\left(1-P_{s}\right){ }^{a P_{s}^{b}}\right) / P_{s}(\mathrm{a}=1.24 \pm 0.15, \mathrm{~b}=-0.41 \pm 0.05)$. Using our simplified description of presynaptic dynamics, we analytically estimated the PPR in our model over a realistic range of basal per-vesicle release probabilities $\left(p_{v}^{0}=10^{-4}\right.$ to 1) and RRP sizes ( $\left.N_{\max }=1-15\right)$, and found the value of $\alpha_{f}$ for which this distribution of values was optimally fit by the above empirical model. The mean-squared error was minimized at $\alpha_{f} \approx 0.03$. We thus set $\alpha_{f}^{*}=0.03$ as the biological reference value of the gain parameter in our simulations, and, separately, also examined the effects of reducing or increasing the level of facilitation on synaptic coding properties (Fig. 1B).

\section{Model implementation}

Realistic values of various parameters for the input stimulus were chosen in accordance with in vivo CA3 spike recordings from awake, freely moving rodents (adult male Long-Evans rats; Fenton and Muller, 1998), which have been used in previous STP studies (Klyachko and Stevens, 2006; Kandaswamy et al., 2010; Rotman et al., 2011). This dataset comprises inhomogeneous spike trains spanning a broad range of discharge frequencies $(\sim 5-60 \mathrm{~Hz})$ and burst sizes $(\sim 3-30$ spikes per burst), with typically long (approximately several seconds) quiescent periods separating individual discharges. We modeled two specific implementations of the temporal signal $\phi(t)$ shaping presynaptic spiking activity: one describing frequency modulation of sporadic PF firing (rate remapping), and another wherein it represents the variable duration (with fixed discharge frequency) of individual PF passes. Non-zero instances of $\phi$ were sampled randomly from an appropriate dynamic range accordingly. For the variable frequency case, spike rate for every pass was sampled from the 6- to $60-\mathrm{Hz}$ range, and the step size was set to $\Delta t=0.5 \mathrm{~s}$, which also gives a consistent range of spike numbers per burst (3-30 on average). Alternately, variable-duration passes were modeled with a fixed in-field firing rate of $30 \mathrm{~Hz}$ (the average discharge frequency from experimental recordings) and individual passes spanning $0.1-1 \mathrm{~s}$, again giving between 3 and 30 spikes per burst on average. Further, to simplify estimation of information rates in this case, the step size was fixed at $\Delta t=0.1 \mathrm{~s}$, and every time a PF pass was reckoned to occur, that step was assigned a variable number of spikes, based on the duration (value of $\phi$ ) corresponding to that instance. Thus, variable duration bursts were rescaled to a constant step $\Delta \mathrm{t}$; this is a valid approximation when estimating average information rates over very long times, provided that burst durations $\ll 1 / r_{s}$, which is compatible with the available data. To reduce errors in estimation of $R_{\text {info }}$ in this approximation, facilitation and refilling time constants were also appropriately rescaled when implementing STP dynamics within every PF crossing by the corresponding duration $t_{B}\left(\tau_{f} \rightarrow \tau_{f} \Delta t / t_{B}\right.$ and $\left.\tau_{r} \rightarrow \tau_{r} \Delta t / t_{B}\right)$, to mitigate 
over- (under-)estimating the effect of activity-dependent facilitation (vesicle recycling).

Monte Carlo simulations of the STP model were conducted for a range of input rates $\left(r_{s}=0.05-0.2 s^{-1}\right)$, low noise levels $\left(r_{n}=0-1 s^{-1}\right)$ and maximum RRP sizes $\left(N_{\max }=1-15\right)$, and dependence of results on the basal $p_{v}^{0}$ was characterized over approximately four orders of magnitude $\left(p_{v}^{0}=0.0001-1\right)$. For every distinct combination of parameter values, 20 independent runs (each of $3 \times 10^{4} \mathrm{~s}$ in duration) were simulated, and the time-averaged rates of information flow and release events were estimated for every trial. The averages from our simulations were found to provide accurate estimates of the asymptotic information rates, justifying comparisons between different synaptic configurations in terms of the corresponding across-trial averages. To specifically assess the differential effect of STF on synaptic function, every synapse with STF (referred to as a dynamic synapse) was compared with an equivalent static synapse which lacks facilitation while still exhibiting activitydependent vesicle depletion (this corresponds to setting $\alpha_{f}=0$ in Eq. 3). In the following, only the results for the model with variable burst frequency are presented, although we have separately verified that the findings are closely reproduced for the variable-duration model as well.

\section{Code accessibility}

All simulations, data analysis, and visualization were performed in Python using the NumPy, SciPy, and Matplotlib modules. Simulations were carried out on a desktop PC (Intel Xeon CPU @ 3.5 GHz × 8, 32 GB RAM) running Ubuntu 16.04 operating system. Basic code implementing the model is freely available online at GitHub (https://github. com/gmcoderepo/stp_at_ca3synapse). The code is available as a Python file in Extended Data 1.

\section{Results}

\section{Improved signaling at unreliable synapses with STF}

How does STF shape the vesicle code conveying information about presynaptic cell activity at stochastic hippocampal synapses? Experimental measurements reveal considerable diversity in the RRP size, release probability and STP properties across individual CA3-CA1 synapses (Dobrunz and Stevens, 1997; Schikorski and Stevens, 1997) that is invoked in our synaptic model (outlined in Materials and Methods and Fig. 1B). To explore the role of various synaptic attributes in modulating its transmission properties, we simulated the synaptic response to regular presynaptic spike trains occurring at different rates. Figure $1 C$ shows the response of a synapse to persistent spiking at $30 \mathrm{~Hz}$ (the average frequency in experimentally recorded bursts) for low and high initial release rates $\left(P_{s}^{0}=\right.$ 0.1 and 0.9 ) and a canonical maximum RRP size of $N_{\max }=$ 8. For the low $P_{s}$ synapse, the time-dependent release probability initially increases because of strong activitydependent facilitation and reaches a maximum, before the effect of vesicle depletion and slower recovery takes over, causing a drop in the response which eventually settles at a steady state value determined by the firing frequency. In contrast, the high $P_{s}$ synapse shows a monotonically decreasing response with time, as it undergoes weaker facilitation and a larger initial $P_{s}$ also implies faster depletion of the readily-releasable vesicle pool. The above qualitative differences illustrate the regimes of synaptic enhancement and depression encompassed by the STP model (Fig. $1 A$ ) that is based on physiological parameters for facilitation and depletion. To further bring out the differences between these two limits, we quantified the asymptotic/steady-state response amplitude of the dynamic synapse to input trains spanning a wide range of frequencies $(0.1-100 \mathrm{~Hz})$ as a function of the initial transmitter release probability. Figure $1 D$ shows the normalized synaptic response profiles for different base synaptic failure rates at a fixed RRP size $\left(N_{\max }=8\right)$. High $P_{s}$ synapses are most effective at transmitting spikes arriving at low frequencies, and with increasing facilitation (lower $P_{s}$ ), the optimal transmission frequency is shifted to higher frequencies, demonstrating a transition from depression-dominated to facilitation-dominated behavior governed by the overall nature of STP dynamics (Eq. 3).

It is to be noted that RRP size also influences synaptic behavior along with the value of $P_{s}^{0}$, the latter being a function of the maximum number of vesicles available for release as well as the basal per-vesicle fusion probability $\left(p_{v}^{0}\right)$. To demonstrate the role of RRP size in modulating synaptic behavior, the frequency-response relation estimated for different numbers of release-ready vesicles with a fixed basal synaptic release rate $\left(P_{s}^{0}=0.2\right)$ is shown in Figure 1E. These curves highlight the role of RRP size in tuning the response profile of the synapse: synapses with a given fixed initial probability of release $\left(P_{s}^{0}\right)$ but differing in their number of available vesicles show a range of responses, from low-pass filtering for smaller $N_{\max }$ (corresponding to high basal $p_{v}^{0}$ ) to higher optimal transmission frequencies for larger RRP sizes (lower basal $p_{v}^{0}$ ). Taken together, the above examples (Fig. $1 C-E$ ) capture the broad repertoire of behavior displayed across an ensemble of facilitating probabilistic synapses in the physiological regime (Dobrunz and Stevens, 1997), that is controlled by the interplay among key synaptic parameters governing transmitter release and recovery.

Naturally occurring firing patterns in the CA3 region are characterized by brief increases in firing frequency (spike bursts) separated by long periods of low activity (Klyachko and Stevens, 2006) and were shown to encode behaviorally relevant integrated spatial and contextual signals. These observations inform our model, and we next examine synaptic processing of spike trains mimicking such activity patterns. Figure $2 A$ illustrates the steps involved in our simulation of STP dynamics for a synapse with $P_{s}^{0}=0.2$ when the presynaptic spiking pattern carries information in the temporal sequence of burst occurrences and the variable firing frequency associated with every burst. The stochastic, time-varying signal (effectively an inhomogeneous Poisson process) is reflected in the brief spike discharges of the presynaptic neuron during passages through its preferred location (PF); this spiking activity drives the temporal dynamics of $p_{v}$ and the transmitter release probability $\left(P_{s}\right)$, eliciting a sequence of vesicular release events. We used a binning procedure to estimate the mean information content in the quantal release profile about the presynaptic signal (for 
A

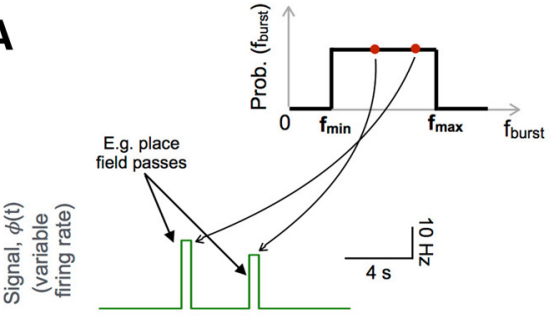

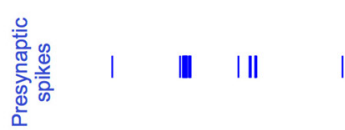
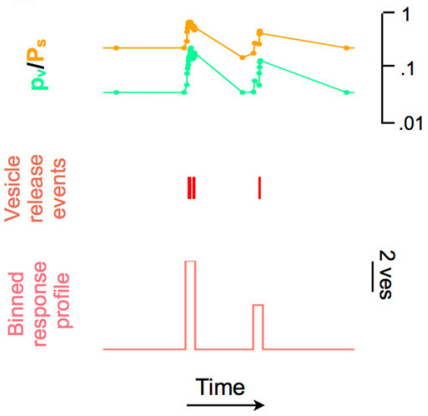

C

Burst frequency $=30 \mathrm{~Hz}$
B

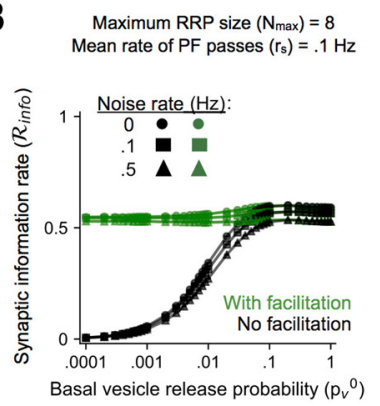

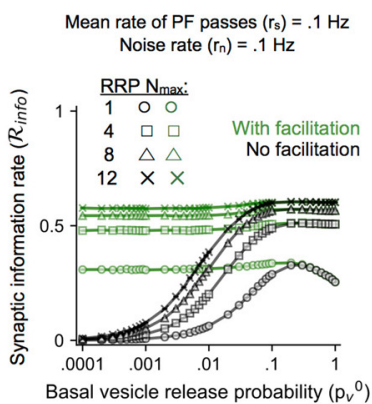

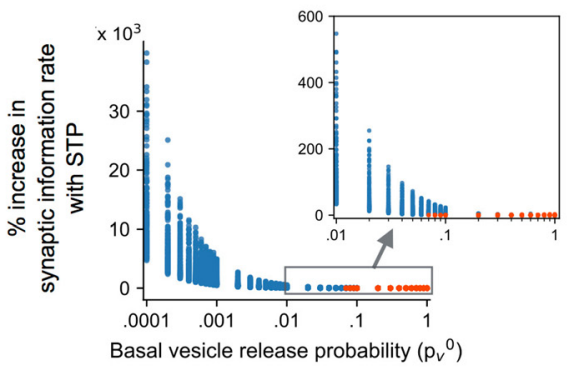

RRP size $\left(\mathrm{N}_{\max }\right)=8$

Initial per-vesicle fusion probability $\left(\mathrm{p}_{\mathrm{v}}{ }^{0}\right)=0.03$

(ii)

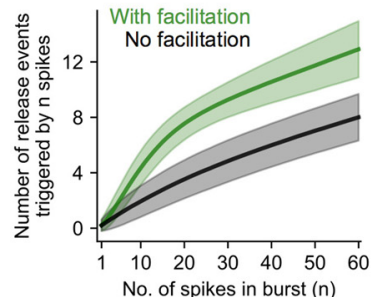

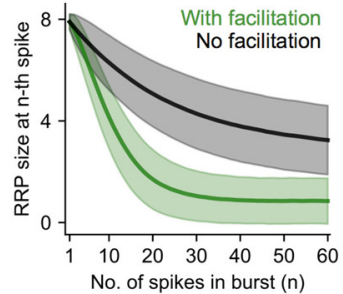

(iii)

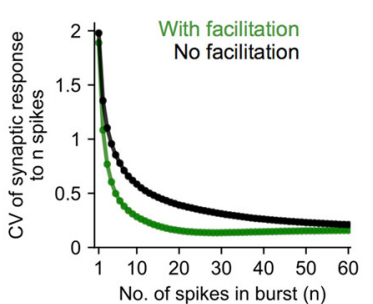

(iv)

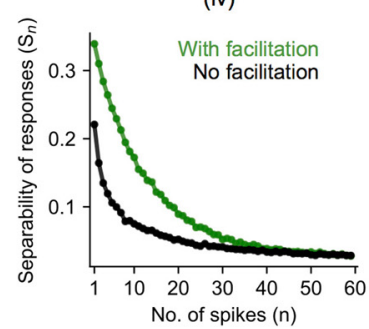

Figure 2. Elevated transmission of presynaptic information with STP of vesicular release. $\boldsymbol{A}$, Time trace illustrating the conversion of an input signal $[\phi(\mathrm{t})]$ to a sequence of synaptic release events governed by STP. For this example, $P_{s}^{0}=0.2, N_{\max }=8$, and $r_{s}=r_{n}=$ $0.1 \mathrm{~s}^{-1}$. The uniform distribution of spiking frequencies from which individual burst instances are randomly sampled is shown in the top right corner. $\boldsymbol{B}$, top left, Time-averaged rate of information carried by synaptic release events $\left(\mathcal{R}_{\text {info }}\right)$ as a function of the basal per-vesicle fusion probability $\left(p_{v}^{0}\right)$ for a synapse with STF (green curves) and an equivalent non-facilitating synapse (black curves). Different markers indicate different noise levels. (Results shown as mean \pm SEM over 20 independent simulations; $N_{\text {max }}=8$ and input rate $r_{s}=0.1 \mathrm{~s}$.) Top right, Synaptic information transfer rates with STP (green curves) and without facilitation (black curves) for different numbers of available vesicles (different markers). (Results shown as mean \pm SEM over 20 independent trials; $r_{n}=r_{s}=0.1$ $s^{-1}$.) Error bars being smaller than the marker size are not visible. Bottom, Enhancement of synaptic information transmission with facilitation summarized as a distribution of relative changes (\% difference of means relative to the static synapse) over a biologically relevant range of input/model parameters (for details, see Materials and Methods). Inset shows a magnified view of the $0.01 \leq p_{v}^{0} \leq$ 1 interval. Color coding indicates statistical significance of pairwise differences (blue: significant at FDR $<0.001$ level, red: not significant). C, Synaptic response (i) and progressive depletion of the readily releasable vesicle pool (ii) as functions of the number of input spikes for a synapse with STF (green) and lacking facilitation (black). Response fluctuations are quantified in terms of the CV of number of release events (iii) and separability of responses to bursts differing in size by a single spike (iv). Results in i, ii shown as mean $\pm \mathrm{SD}$ (1000 independent trials); $P_{s}^{0}=0.2, N_{\max }=8$, and spikes are Poisson-distributed with mean frequency of $30 \mathrm{~Hz}$.

details, see Materials and Methods). Figure $2 B$, top left, shows the relative mutual information rate, $\mathcal{R}_{\text {info }} \equiv \mathcal{R}_{r s} / \mathcal{R}_{s}$ as a function of the basal probability of vesicle release $\left(p_{v}^{0}\right)$ for different noise levels, for both a canonical synapse exhibiting STP (green lines) and an equivalent synapse which does not show facilitation (black lines). Figure $2 B$, top right, illustrates the dependence of synaptic information rates on the RRP size for a fixed noise level of $r_{n}=0.1 \mathrm{~Hz}$. These examples indicate a general enhancement of synaptic information transfer with STF, significantly expanding the scope of previous results from spike-based studies (Pfister et al., 2010; Rotman et al., 2011). Further, this increase is more pronounced for synapses with lower release probability, aligning with our expectation that smaller basal $p_{v}^{0}$ combined with stronger facilitation (Fig. $1 B$ ) accentuates the differential response to bursts versus single spikes, enhancing the ability of the synapse to selectively transmit information-carrying brief, high-frequency spike discharges. To address the generality of the above 
results, we repeated our simulations over a biologically relevant range of parameter values (input rate/mean rate of $\mathrm{PF}$ passes $=0.05-0.2 \mathrm{~Hz}$, noise level $=0-1 \mathrm{~Hz}$ and RRP size $=1-$ 15). The overall difference in synaptic information capacity in the presence and absence of STF is summarized as a distribution of relative changes in $\mathcal{R}_{\text {info }}$ (percent difference of means) in Figure $2 B$, bottom, and the color coding represents statistical significance of pairwise differences [two-sided Wilcoxon rank-sum test followed by Benjamini-Hochberg adjustment for multiple comparisons; blue: significant at false discovery rate (FDR) $<0.001$, red: not significant]. STF is found to robustly improve the fidelity of synaptic signaling in the physiological regime. The differential effect of facilitation scales inversely with the basal synaptic release efficacy, and is more marked for higher synaptic failure rates (lower $p_{v}^{0}$ ). The effect of facilitation is diminished with increase in the basal release probability, and there is little difference in transmission efficacy between the dynamic and static synapses for $p_{v}^{0}$ values above $\sim 0.1$.

How can this improved reliability of synaptic information transmission be understood in simple terms? We recall that the synaptic response to information-carrying brief spike discharges consists of a variable number of release events; thus, the information content of the vesicle code in our formulation is essentially determined by how well the different output sizes (total number of releases triggered by a spike discharge) can discriminate between different input states (i.e., the variable spiking frequency, or duration, associated with every burst). We characterize the reliability of this mapping in terms of the cumulative number of released vesicles as a function of the burst size (number of spikes). Figure $2 \mathrm{Ci}$ compares the responses of a canonical synapse (basal $p_{v}^{0}=0.03$ and maximum RRP size $=8$ ) with and without STF to Poisson spiking at a mean rate of $30 \mathrm{~Hz}$. In the presence of facilitation, not only is the average response amplitude (total number of release events for a given burst size) larger, but more importantly, it is also a more reliable readout of the burst size, because of reduced variability of responses relative to the static synapse. This difference is clearly seen in Figure 2Ciii, iv, where two distinct measures for response fluctuations are plotted as functions of the number of spikes $(n)$. The coefficient of variation (CV; defined as the standard deviation of the response relative to its mean) is lower for the synapse with facilitation, and the separability, defined as $S_{n}=\left(\mu_{n+1}-\mu_{n}\right) /\left(\sigma_{n+1}+\sigma_{n}\right)\left(\mu_{n}\right.$ and $\sigma_{n}$ denoting the mean and SD, respectively, of the total response to $n$ spikes), is correspondingly larger. The initial steeper increase in the mean response with the number of spikes (Fig. 2Ci), together with smaller dispersion of responses (Fig. 2Ciii,iv), implies better correspondence between the response amplitude and the burst length when STF is included. It is to be noted, though, that this increased reliability is also accompanied by faster depletion of the readily-releasable vesicle pool at the facilitating synapse (Fig. 2Cii), implying a reduced dynamic range of burst sizes that can be conveyed by synaptic release events (this is indicated by a sharp change in the slope of the response profile for the facilitating synapse beyond some threshold spike number in Fig. 2Ci). However, our results indicate that, in the biologically relevant parameter range considered here, this downside of STP is more than offset by the advantage of increased reliability of responses to the shorter bursts (Fig. 2Ciii,iv) in the presence of facilitation, leading to significant net improvement in synaptic information transfer (Fig. 2B). In sum, our simulations of STP dynamics highlight a crucial functional role for activity-dependent facilitation at unreliable hippocampal synapses, in conferring distinct advantage for the transmission of information represented by time-varying presynaptic cell activity in a physiologically relevant setting.

\section{Reliable signaling at realistic STP synapses is nearly independent of their basal release properties}

The results in the previous section (Fig. 2B) indicate that, for realistic number of vesicles, the signaling capacity of an STP synapse is not only increased relative to a static synapse, but, notably, also independent of its initial per-vesicle release rate $\left(p_{v}^{0}\right)$ for the characteristic inhomogeneous spiking patterns associated with CA3 pyramidal cells studied here. To elaborate on the dependence of synaptic information transfer on its basal transmission probability, we scaled the mean information rate estimated for each value of $p_{v}^{0}$ by the maximum value attained across the full range of $p_{v}^{0}$ values considered (this was done separately for every combination of input rate, noise rate, and vesicle pool size). This rescaling factors out the dependence on the other model parameters, and reveals the general trend in dependence of synaptic information transfer on its intrinsic reliability.

Figure $3 A$, top, shows the distribution of rescaled information capacity values separately for synapses with STF (green points) and lacking facilitation (gray points); each point represents a particular combination of model parameter values and $p_{v}^{0}$. These results indicate that synaptic information coding is robust to differences in the basal $p_{v}$ at dynamic synapses. In other words, STP ensures that synapses with widely different basal fusion probabilities transmit at comparable rates (median of values for each $p_{v}^{0} \gtrsim 95 \%$ over the full range of $p_{v}^{0}$ values considered here). By contrast, information carried by synapses lacking facilitation shows clear dependence on the magnitude of the basal $p_{v}$, and is strongly impaired for synapses with $p_{v}^{0} \lesssim 0.1$.

These data are especially interesting in the light of the considerable heterogeneity in the magnitude of $p_{v}^{0}$ at hippocampal CA3 synaptic populations as reported by experimental studies (Dobrunz and Stevens, 1997; Murthy et al., 1997; Holderith et al., 2012). Our analysis indicates that synaptic information transfer in the presence of dynamic gain control (Eq. 3) is nearly invariant to differences in the basal fusion probability per vesicle (Fig. $3 A$, bottom), and there is no appreciable change in the overall information transfer profile over nearly four orders of $p_{v}^{0}$ (Fig. 3A, top). Recalling that the information rate (Eq. 2) provides a measure of how well the different presynaptic input states (spiking frequencies) are discriminated by the output states (total transmitter release per spike burst), the form of STP (Eq. 3) with physiological facilitation strength is such that low release probability synapses 
A

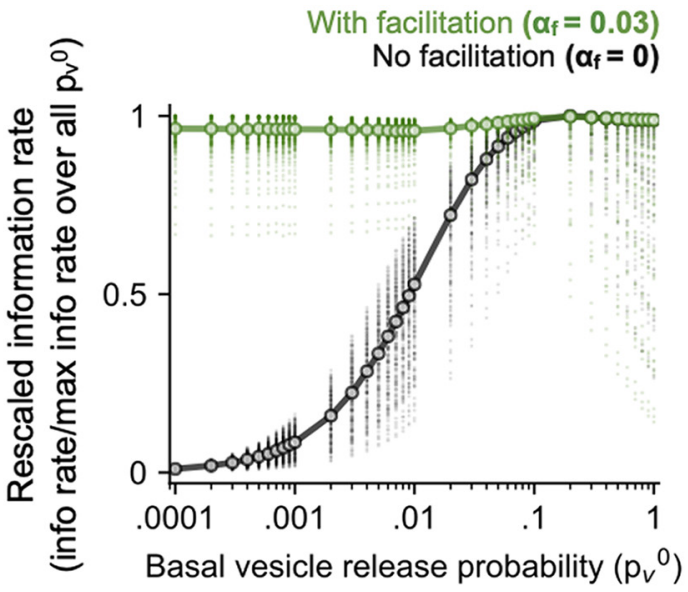

B

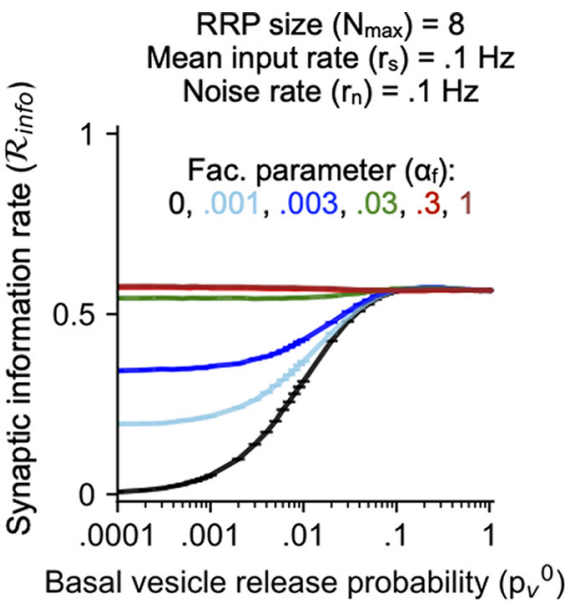

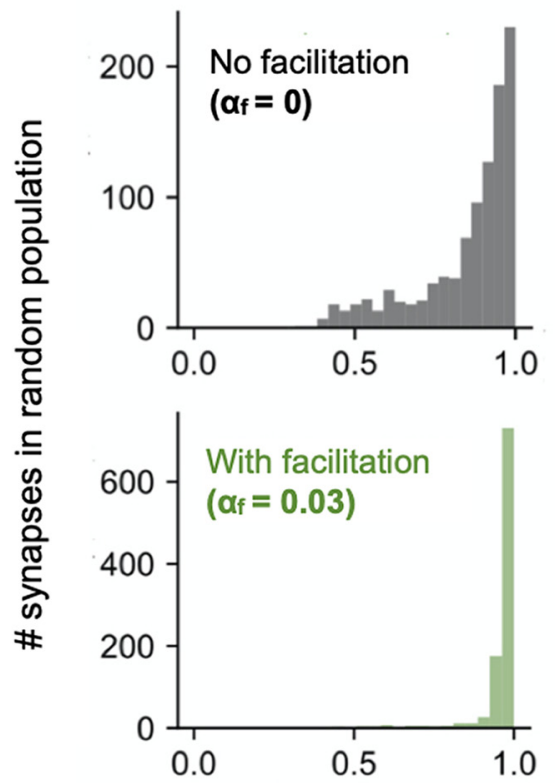

C

Facilitation parameter $\left(\alpha_{\mathrm{f}}\right)$ :

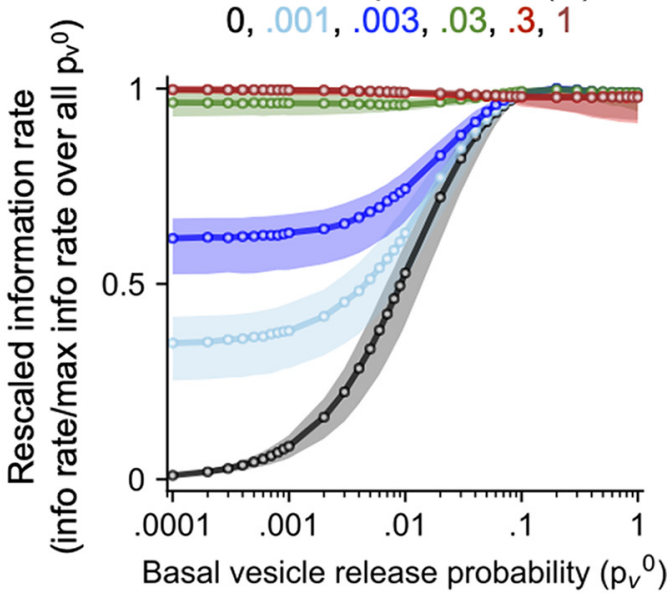

Rescaled information rate (info rate/max info rate over all $\mathrm{p}_{\mathrm{v}}{ }^{0}$ )

Figure 3. Information transmission properties in an ensemble of facilitating CA3-CA1 synapses. $\boldsymbol{A}$, top, Dependence of information rate estimates (rescaled values) on the basal probability of vesicle release $\left(p_{v}^{0}\right)$ for synapses with physiological facilitation strength (green) and non-facilitating synapses (black) over a realistic range of input/model parameters (for details, see Materials and Methods). Every point represents a distinct parameter combination, and continuous lines connect the medians (one per value of $p_{v}^{0}$ ). Bottom, Distribution of rescaled information rates in a representative population of static (black) and facilitating (green) synapses with variable per-vesicle basal release probability $\left(p_{v}^{0} ; n=1000\right.$ synapses, randomly sampled from $0.05 \leq P_{s}^{0} \leq 0.6$ and $1 \leq N_{\text {max }} \leq$ 15 ; input rate and noise rate were also randomly set for each synapse). $\boldsymbol{B}$, Estimated time-averaged information transfer rate as a function of the initial per-vesicle release probability $\left(p_{v}^{0}\right)$ for synapses with different levels of facilitation (approximately three decades in the facilitation parameter $\left.\alpha_{f}\right)$. Results shown as mean \pm SEM (20 independent trials) for each choice of $\alpha_{f}$. $N_{\text {max }}=8$; rate of burst occurrences $=$ noise rate $=0.1 \mathrm{~s}^{-1}$. The non-facilitating static synapse is shown in black. $\boldsymbol{C}$, Distributions of rescaled information rates over a realistic range of inputs/model parameters for different magnitudes of the synaptic gain $\alpha_{f}(0.001-1)$. Each distribution is displayed in terms of the medians and 25th -75 th percentile (interquartile) ranges (per value of $p_{v}^{0}$ ).

achieve a discriminability comparable to synapses with more reliable release. Although we have demonstrated this for a specific dynamic range of CA3 spiking frequencies consistent with available experimental data, the invariance of synaptic information capacity to the basal release probability is found to hold fairly robustly over a range of input distributions [maximum burst frequency $\left(f_{\max }\right) \gtrsim 40 \mathrm{~Hz}$; data not shown]. Thus, we propose that physiologically realistic STP works to counteract degradation of presynaptic signals at synapses with small release probabilities, and enables CA3-CA1 synapses to maintain stable information rates in the face of necessary heterogeneity in the basal $p_{v}$, arising from long-term changes associated with learning 
or homeostatic plasticity mechanisms on the cellular/network level.

The above analysis reveals significant overall difference in the profile of information transfer at stochastic synapses in the presence of STF (Fig. 3A). How sensitive are these effects to its magnitude? Recalling that the dynamics of the release probability in our effective description of STP (Eq. 3) is essentially controlled by the gain parameter $\alpha_{f}$, which was adjusted to be compatible with experimental findings, we ask how the behavior of synapses changes for weaker or stronger facilitation. Figure $3 B$ shows an example of the (unscaled) synaptic information rate (mean \pm SEM) as a function of the basal $p_{v}^{0}$ for a synapse with $N_{\max }=8$ vesicles, across a range of $\alpha_{f}$ values spanning approximately three orders of magnitude $(0.001-1)$. Figure $3 C$ compares the distributions of the rescaled information rate (estimated as before over a broad range of model parameters) for different facilitation strengths. The dispersion of estimates for each $p_{v}^{0}$ is represented in terms of the median (solid line) \pm interquartile range (IQR) separately for every $\alpha_{f}$. These results indicate that the profile of scaled synaptic information capacity is strongly modulated by changes in $\alpha_{f}$ especially at the smaller $p_{v}^{0}$ values $(\lesssim 0.1)$. This is a reflection of the greater sensitivity of facilitation at smaller basal release probabilities to changes in $\alpha_{f}$ in the STF model (Fig. $1 B$ ). In particular, reduction of $\alpha_{f}$ below the biological estimate $\left(\alpha_{f}^{*}\right)$ suppresses information transfer for smaller release probabilities and introduces heterogeneity in the ensemble behavior, whereas for $\alpha_{f} \gtrsim \alpha_{f}^{*}$, the rescaled information capacity is nearly independent of the basal synaptic failure rate.

\section{Short-term release dynamics regulates the capacity- cost trade-off at probabilistic synapses}

In the previous section, we showed that STF, in general, enables probabilistic synapses to signal the occurrence and length of brief high-frequency spike discharges more reliably. What is the theoretical limit on synaptic information capacity achievable at individual facilitating synapses, when transmitter release is governed by the STP model analyzed here (Fig. 1A)? For every combination of stimulus rate $\left(r_{s}\right)$, noise $\left(r_{n}\right)$, and RRP size $\left(N_{\max }\right)$, we estimated the maximum rate of synaptic information transfer attainable when $\alpha_{f}$ and $p_{v}^{0}$ are allowed to vary, and we examined how well biological synapses (corresponding to $\alpha_{f}=\alpha_{f}^{*}$ ) compare against this upper bound on $\mathcal{R}_{\text {info }}$ (denoted as $\mathcal{R}_{\text {info }}^{*}$ ). Figure $4 A$ displays the distributions of the normalized channel capacity $\left(\mathcal{R}_{\text {info }} / \mathcal{R}_{\text {info }}^{*}\right)$ for different choices of $\alpha_{f}$ (different colors); for every $p_{v}^{0}$, the distribution over a range of input parameters and RRP sizes is represented in terms of the median and IQR. Our results show that biological synapses $\left(\alpha_{f} \approx \alpha_{f}^{*}\right)$ uniformly reach high, near-optimal, information rates under physiological conditions over approximately four orders of magnitude of the basal per-vesicle release probability examined here (the median of normalized estimates for each $p_{v}^{0}$ exceeds $90 \%$ over the full range of $p_{v}^{0}$ values considered). By contrast, probabilistic synapses with weaker facilitation (by a factor of 10 relative to the physiological level), or no facilitation altogether, are much less effective at conveying information about presynaptic spiking activity, and the fidelity of information transfer at these synapses is markedly suppressed for $p_{v}^{0} \lesssim 0.1$ (Fig. $4 A$ ).

Previous studies have emphasized the relevance of energetic constraints for a better understanding of neurobiological design on diverse scales (Laughlin, 2001; Laughlin and Sejnowski, 2003); examples from sensory systems, in particular, suggest that synaptic function may be significantly influenced by energy (resource) limitations (Laughlin et al., 1998; Harris et al., 2015). To evaluate the potential role of energy constraints in shaping synaptic information processing in the hippocampus, we revisit the example in Figure $3 B$, and quantify the synaptic resource use vis-à-vis information transfer at individual facilitating synapses. Figure $4 B$ shows the dependence of the average vesicular release rate and the energy efficiency of information transduction ( average number of vesicles needed to transmit a bit), respectively, on the basal $p_{v}$ for different levels of synaptic facilitation (different colors) at a canonical CA3 synapse $\left(N_{\max }=8\right)$. In general, energy use scales up with the basal probability of release $\left(p_{v}^{0}\right)$ and with the strength of synaptic facilitation $\left(\alpha_{f}\right)$, as expected (Fig. $4 B$, left). Notably, though, an increase in synaptic information transfer with stronger facilitation is accompanied by reduction in the synaptic energy efficiency, i.e., each released vesicle packs a smaller punch on average (Fig. $4 B$, right). The supralinear scaling of energy costs with synaptic information capacity implied by these examples suggests that in the context of realistic spiking patterns, individual CA3 synapses do not operate at optimal energy efficiency (according to the local measure of efficiency examined here), or minimize energy consumption; in fact, synapses lacking STP (Fig. 4B, black curves) require fewer releases per unit of information transmitted, albeit at significantly reduced overall information capacity, relative to dynamic synapses.

Do energy constraints, then, play no significant role in shaping the vesicle code at probabilistic hippocampal synapses? Examining the regime of stronger facilitation $\left(\alpha_{f} \gtrsim \alpha_{f}^{*}\right)$ in the above example provides a potential clue in this regard. Figures $3 B, 4 B$ together indicate that a canonical synapse operating in the physiological range $\left(\alpha_{f}\right.$ $\approx \alpha_{f}^{*}$ ) transmits information at near-optimal capacity, and further increase in $\alpha_{f}$ (by one order of magnitude, from 0.03 to 0.3 or 1 ) provides little additional benefit; the increased facilitation is, however, accompanied by a disproportionately larger increase in energy costs of synaptic transmission, which may be seen by comparing the green with the red/brown curves separately in Figures $3 B, 4 B$. This specific example suggests that biological CA3 synapses may be poised to operate near the upper bound on information transfer rate while energy usage is economized to the extent that performance is not compromised.

To elaborate on the nature and generality of this energy-function trade-off, we compared biological STP synapses $\left(\alpha_{f} \sim \alpha_{f}^{*}\right)$ with synapses exhibiting weaker or stronger facilitation over approximately three decades of magnitude, estimating the relative change in the mean 


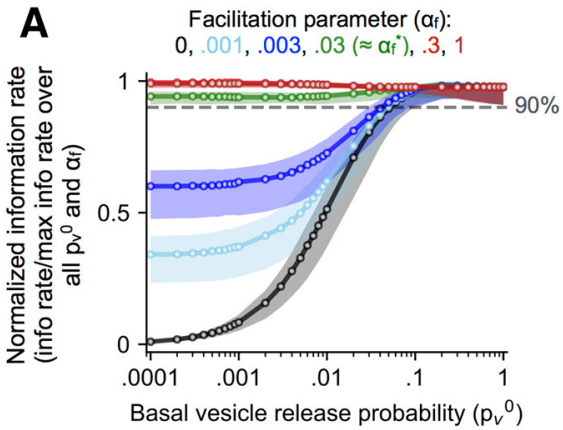

C

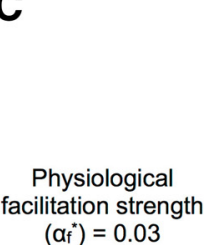

$\left(\boldsymbol{\alpha}_{\mathrm{f}}^{*}\right)=0.03$

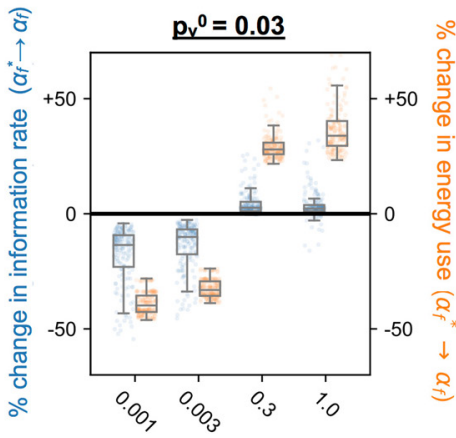

Facilitation parameter $a_{f}$

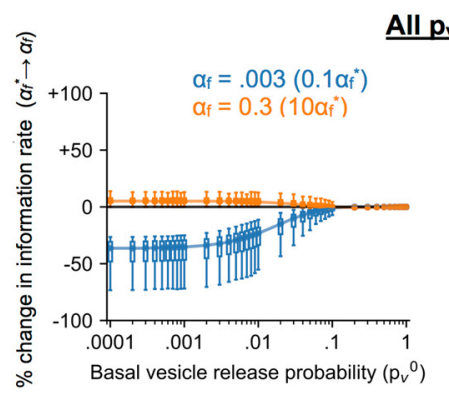

All $\mathbf{p}^{0}$

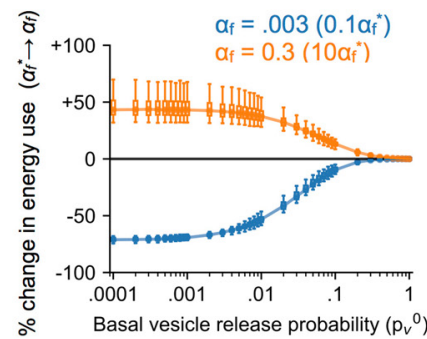

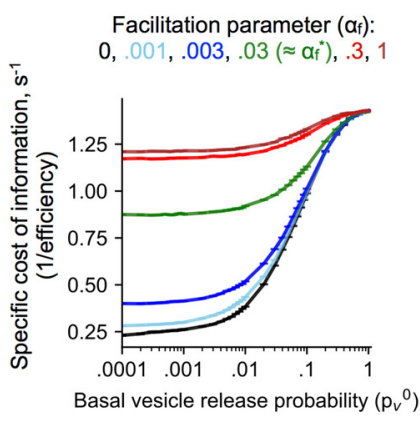

D

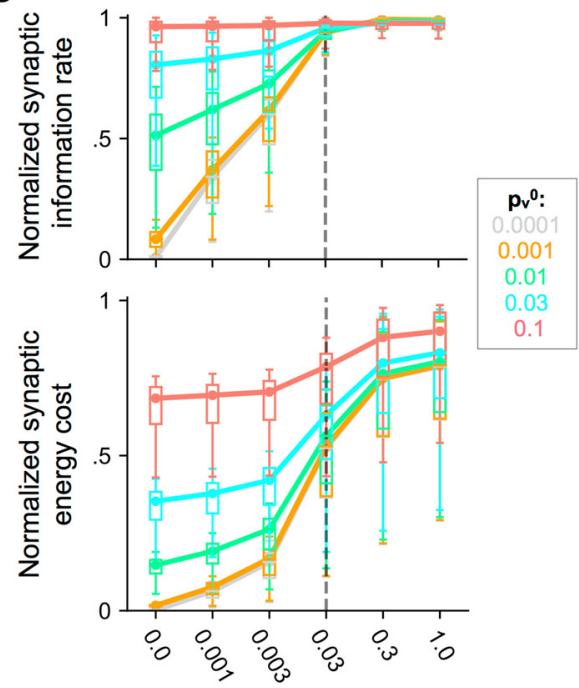

Gain parameter $\alpha_{f}$

Figure 4. Optimal signaling and synaptic energy efficiency with physiologically realistic STP dynamics. $\boldsymbol{A}$, Distributions of normalized synaptic information rate (fraction of maximum capacity) over a biologically relevant range of parameters for different choices of the facilitation parameter $\alpha_{f}$ (different colors). Each distribution is displayed in terms of the data medians and IQRs over a broad range of $p_{v}^{0}$ values. Realistic synapses $\left(\alpha_{f}^{*} \approx 0.03\right)$ transmit at close to maximum capacity overall (median values $>90 \%$ across all $\left.p_{v}^{0}\right)$. B , Example profiles of the time-averaged energy usage (left) and the specific cost of information transfer, i.e., inverse of the efficiency (right) for synapses with different facilitation strengths (all other parameter settings are same as in the example in Fig. 3B). Data shown as mean \pm SEM (20 independent simulations). $\boldsymbol{C}$, top, Box-plots of relative changes (\%) in synaptic information capacity and energy requirement when the synaptic gain is scaled either up or down by a factor of $\sim 10$ relative to its physiological level $\left(\alpha_{f}^{*}\right)$ for a synapse with basal $p_{v}^{0}=0.03$. Each distribution covers a biologically relevant range of input parameters and maximum RRP sizes ( $n=180$ points; for details, see Materials and Methods). Bottom, Summary statistics of relative changes (\%) in synaptic information rate (left) and average frequency of release events (right) when $\alpha_{f}$ is scaled up (orange) or down (blue) by $10 \times$, for a wide range of $p_{v}^{0}$ values. $\boldsymbol{D}$, Estimates of normalized information capacity (top) and normalized energy expenditure (bottom) as functions of the synaptic facilitation strength $\alpha_{f}$ which spans approximately three orders of magnitude (also shown, for reference, are results for the static synapse, corresponding to $\alpha_{f}=0$ ). Each box summarizes the results over a biologically relevant range of input/model parameters, and lines connect the median values for each choice of $\alpha_{f}$; profiles for different choices of the basal $p_{v}^{0}$ are represented by different colors. Vertical dashed lines highlight the biological operating point $\left(\alpha_{f}^{*} \approx 0.03\right)$.

signaling capacity and mean energy cost per synapse when $\alpha_{f}$ is scaled up or down by a factor of $\sim 10$ relative to its physiological reference value $\left(\alpha_{f}^{*}\right)$. Figure $4 C$, top, shows the distribution of relative changes over a range of model parameters (see Materials and Methods) for the specific example of $p_{v}^{0}=0.03$, and each cluster of data points represents a different comparison $\left(\alpha_{f}^{*} \rightarrow \alpha_{f}\right)$. This aggregated data from our simulations indicates that stronger synaptic facilitation relative to the biological setpoint provides little improvement in information transfer rates, but a relatively larger increase in energy expenditure; reducing facilitation, on the other hand, is associated with a sharp reduction in synaptic information capacity. The overall differences evident in Figure 4C, top, are found to be quite general and hold across a broad range of $p_{v}^{0}$ values examined (Fig. $4 C$, bottom). 
The general trends suggested by Figure $4 C$ are brought out clearly in Figure $4 D$, which shows how the normalized synaptic information capacity $\left(\mathcal{R}_{\text {info }} / \mathcal{R}_{\text {info }}^{*}\right)$ and normalized average frequency of release events $\left(\mathcal{R}_{\text {ves }} / \mathcal{R}_{\text {ves }}^{*}\right)$ vary with the strength of synaptic facilitation $\left(\alpha_{f}\right)$. Each box (median \pm IQR) summarizes the distribution of values for a particu$\operatorname{lar} p_{v}^{0}$, and the different colored lines connect the median values corresponding to each choice of $p_{v}^{0}$. Simulations of our STP model suggest that synaptic information capacity is in general an increasing function of the strength of facilitation $\left(\alpha_{f}\right)$, but saturates around the physiological level ( $\alpha_{f} \approx \alpha_{f}^{*}$; Fig. $4 D$, top). Comparing it to Figure $4 D$, bottom, further increase in synaptic gain comes at a larger energy cost, bringing diminishing returns. By contrast, reducing facilitation below the biological operating point $\left(\alpha_{f} \approx \alpha_{f}^{*}\right)$ by approximately one order of magnitude compromises synaptic channel capacity considerably, and the suppression of information transfer rates is particularly marked at smaller vesicular release probabilities $\left(p_{v}^{0}\right)$. In sum, our results quantitatively demonstrate a novel form of local optimization embodied by STP of vesicular release at probabilistic CA3-CA1 synapses, and suggest that, under physiological conditions, individual synapses do not consume more resources than necessary while supporting highest-possible fidelity of information transmission over a wide range of presynaptic strengths.

\section{Discussion}

Does an evolutionary drive toward energy-efficient signaling provide a relevant design principle to account for the salient properties of probabilistic transmitter release at individual hippocampal synapses? Previous investigations have focused on understanding energetic optimality at sensory pathway synapses (Laughlin et al., 1998; Harris et al., 2015, 2019; James et al., 2019). Given the diversity in synaptic morphology and tight structure-function relationships in synapses observed across brain areas, questions on synaptic design must be specific and addressed in a local context. In line with this, we examined STP at a cortical facilitating synapse, specifically the hippocampal Schaffer collateral-CA1 connection. Our synaptic model invoked detailed characterizing properties of single CA3 presynaptic terminals such as RRP size, release probability per vesicle and facilitation profiles derived from experiments and evaluated their impact on gating of realistic activity patterns. This allowed us to obtain biologically relevant insights into synapse-specific transmission properties in the hippocampus. Our results potentially suggest a normative account of biologically observed synaptic facilitation in terms of a local trade-off between two fundamental design constraints, vesicular information coding and energy utilization.

We estimated the capacity of a dynamic synapse, viewed as an unreliable channel, to relay behaviorally relevant temporal signals coded in presynaptic spiking activity via discrete vesicular release events. Our quantitative analysis shows how STF significantly improves the fidelity of synaptic information transduction. Remarkably, our simulations demonstrate that realistic STP enables vesicular release with widely different basal failure rates $\left(p_{v}^{0}\right)$ to convey brief, variable high-frequency spike bursts with comparable fidelity; notably, this invariance is absent at static or weakly facilitating synapses, and is also not found in a phenomenological STP model used previously (Tsodyks and Markram, 1997; Pfister et al., 2010), highlighting the importance of incorporating synapse-specific experimental data to arrive at physiologically relevant findings about synaptic function. Further, physiological information rates over a broad range of release probabilities closely approach the predicted maximum capacity of a facilitating synapse of this type, i.e., within the limits imposed by the overall form of the model of presynaptic dynamics analyzed here. We estimated synaptic energy expenditure in terms of the average quantal release rate; this definition has been used previously to address energy efficiency of information processing in other neural contexts at both the synaptic and cellular levels (Levy and Baxter, 2002; Goldman, 2004; Harris et al., 2012; James et al., 2019). The metabolic cost of transmission at chemical synapses is primarily accounted for by pumps that reset the postsynaptic membrane potential and calcium transients to their resting values; additional but relatively smaller demands are made by reuptake of released glutamate from the synaptic cleft via the action of surrounding astrocytic glutamate transporters, and by the endocytic machinery involved in transmitter vesicle recycling and replacement at the presynaptic terminal (Harris et al., 2012). All of these energy-requiring processes scale up in direct proportion to the number of vesicular releases occurring, hence, the vesicle use, i.e., mean rate of release events, provides an accurate, equivalent measure of the net synaptic energy cost.

Altogether, our results suggest a nuanced form of optimality that is at odds with maximization of synaptic efficiency (average number of quanta released per bit transmitted). Instead, our findings are consistent with the view that realistic STP synapses are poised, to within one order of magnitude in the gain parameter $\alpha_{f}$, to maintain near-maximal information transmission rates while penalizing excessive energy use (Fig. 5). Thus, we present evidence that energetic costs may also be important for regulating the properties of short-lived facilitation at lowrelease probability synapses. Interestingly, an analogous form of optimality was previously proposed in the context of the mammalian visual system (Vincent and Baddeley, 2003). Here, it was shown that synaptic energy restrictions can significantly shape early stimulus representations in the retina, and that the observed center-surround neural receptive fields provide the best balance between efficiency and performance, enabling near-maximal information transmission with largest possible synaptic energy savings. It remains to be seen, to what extent our findings are relevant to some of the other facilitating synapses in the mammalian central nervous system (Atluri and Regehr, 1996; Henze et al., 2002).

A key insight from our model is that synaptic information rates with physiological STP are nearly invariant to differences in the basal fusion probability per vesicle $\left(p_{v}^{0}\right)$ that are present among individual CA3-CA1 synapses. This synaptic diversity, on the one hand, may represent 


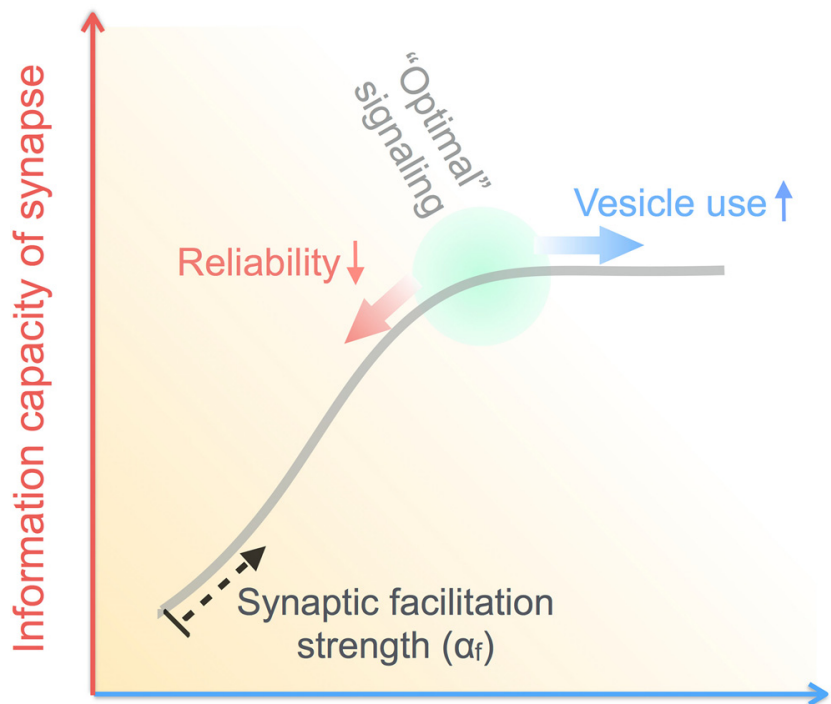

\section{Energy cost of synapse}

Figure 5. Activity-dependent STF regulates the cost-versus-capacity trade-off at unreliable CA3-CA1 synapses. The feasible "configurational space" of an STP synapse (schematically represented by the gray curve) is parametrized by the strength of synaptic gain, which constrains the relation between information transmitted across the synapse and the corresponding usage of synaptic resources. Our results suggest that biological synapses localize to the optimal set-point indicated in green.

the intrinsic, across-synapse differences in the ultrastructural details regulating transmitter release (Atwood and Karunanithi, 2002). On the other hand, a heterogeneous distribution of release probabilities may be a reflection of synaptically encoded memories, which are thought to be stored as distributed patterns of synaptic strength changes via activity-dependent long-term plasticity (McNaughton and Morris, 1987; Moser et al., 1998). Experimental evidence, besides theoretical considerations, suggests that both Hebbian and heterosynaptic plasticity in the hippocampus can have a presynaptic as well as postsynaptic locus of expression (Stevens and Wang, 1994; Enoki et al., 2009; Bliss and Collingridge, 2013; Letellier et al., 2016; Costa et al., 2017), which may, in part, be instantiated as persistent changes in $p_{v}^{0}$. Additionally, variation in presynaptic strengths may arise as a consequence of homeostatic (Branco et al., 2008; Zhao et al., 2011; Davis and Müller, 2015; Soares et al., 2017) or neuromodulatory (Qian and Saggau, 1997; Fernández de Sevilla and Buño, 2003) regulation of presynaptic calcium influx. In summary, several ongoing processes likely underlie the observed dispersion in CA3 presynaptic efficacies. Our analysis suggests that realistic STP dynamics operates at a set-point that compensates for this synaptic heterogeneity to support stable information rates. This implies a possible mechanism to insulate the dynamic synaptic interactions shaping short-timescale information processing of behaviorally evoked activity in the hippocampal circuit from the slower, longer-term adaptive changes that may be happening at these synapses because of learning or homeostatic adjustments. However, STP does not preclude other functional effects of enduring changes in synaptic efficacy, which can, e.g., continue to crucially affect retrieval of stored network patterns through associative recall, promote replay of activity sequences generated during navigational tasks that contributes to spatial memory consolidation, or govern the homeostatic regulation of neuronal activity levels. While the focus of our work is the presynaptic terminal, the long-term up or down-regulation of transmission at Schaffer collaterals can, in fact, occur postsynaptically as well, as shown by numerous experimental studies (Malenka and Bear, 2004); the postsynaptic changes, not explicitly considered here, would not directly impact our analysis of information capacity of presynaptic transmitter release. Overall, our findings add to the functional repertoire of short-term synaptic dynamics, which complements longer-lasting plasticity mechanisms to significantly enhance the functional complexity of biological synapses.

The use of synaptic resources versus the information transmitted is constrained by the synaptic gain parameter $\alpha_{f}$, which essentially controls the steepness of the facilitation profile for low release probabilities (Fig. $1 B$, left) and decides the operating point of the ensemble of CA3 synapses (Figs. 4D, 5). $\alpha_{f}$ may be tuned over evolutionary timescales to some suitable optimum determined by the relative influence of different, competing selective pressures. This aligns with recent understanding of the evolutionary diversification of the synaptic proteome that may have contributed to functional specializations in brain areas and emergent behavior (Emes and Grant, 2012). In the context of the biophysical machinery governing transmitter release, what does the parameter $\alpha_{f}$ correspond to? The basal probability of spike-evoked release is governed by the synchronous activation of the fast calcium sensor, Synaptotagmin-1 (Syt1; Geppert et al., 1994; Fernández-Chacón et al., 2001). On the other hand, recent findings have identified a separate calcium sensor, Syt7, carrying a high-affinity binding site for $\mathrm{Ca}^{2+}$ but with relatively slower kinetics (Bacaj et al., 2013), which was shown to be essential for progressive synaptic facilitation at CA3CA1 terminals during persistent stimulation but not for the initial (basal) synaptic response (Jackman et al., 2016). Efficacy of its interaction with the protein machinery mediating vesicle fusion, or the kinetic parameters governing its sensitivity to calcium, could thus provide a possible biophysical basis to interpret the parameter $\alpha_{f}$. Alternately, kinetic parameters regulating calcium-induced calcium release from intracellular stores which has been implicated in enabling STF at hippocampal synapses (Emptage et al., 2001; Zhang et al., 2009), or developmental parameters regulating the relative arrangement of calcium channels and the release machinery (Nadkarni et al., 2012; Vyleta and Jonas, 2014), may determine the magnitude of $\alpha_{f}$. Biophysically detailed computational models of presynaptic calcium dynamics (Nadkarni et al., 2010; Hamid et al., 2019), outside the scope of the present study, can potentially shed more light on the molecular underpinnings of STP approximated by the reduced description in Equation 3 and help suggest mechanistic interpretations of $\alpha_{f}$.

Although our study specifically examines the role of local constraints in shaping synaptic release properties, it 
is expected that synapse design also carries imprints of selection pressures at higher levels of neural organization. A number of previous studies have elaborated on the functional implications of synaptic STP for collective dynamics on neuronal networks (Levina et al., 2007; Mongillo et al., 2008; Mejias and Torres, 2009). It is thus plausible that properties of individual synapses reflect such system-level design considerations as well. The present work, in particular, does not account for the typical RRP size of CA3 synapses, which is experimentally found to be close to $\sim 10$ vesicles per bouton (Dobrunz and Stevens, 1997; Schikorski and Stevens, 1997). Our analysis, in fact, indicates that average synaptic information capacity is a monotonically increasing function of the size of the RRP; thus, if information transfer is to be improved, larger synapses ought be favored, which runs somewhat counter to the limit on synapse size reported by experiments. We surmise that an optimal RRP size might represent a compromise between reliability of signaling at individual synapses and information processing capacity on the network level. Given strong constraints on neural volume (or equivalently, on total availability of synaptic resources) as proposed previously (Laughlin and Sejnowski, 2003; Varshney et al., 2006), cortical connectivity might trade off high-fidelity synaptic transmission (proportional to the RRP size) for increased network complexity from a higher density of smaller, albeit less reliable, synapses (based on scaling arguments; Newman, 1988; Chklovskii et al., 2002). Detailed analysis of network information processing under physical constraints will be needed to evaluate the role of such an interaction across scales in shaping the design of fundamental processing elements in the brain.

To conclude, we propose that quantitative properties of probabilistic vesicular release at individual hippocampal synapses can be meaningfully interpreted in terms of a local cost-versus-capacity trade-off. The present analysis for a synapse in a higher brain circuit crucial to learning resonates with a growing body of work on the generality of information processing principles in understanding diverse cellular processes (Tkačik and Bialek, 2016). Our results suggest that the design of single synapses is primarily adapted to ensure optimal performance for diverse synaptic strengths, and this is achieved in an energetically cost-effective manner.

\section{References}

Abbott LF, Regehr WG (2004) Synaptic computation. Nature 431:796-803.

Allen C, Stevens CF (1994) An evaluation of causes for unreliability of synaptic transmission. Proc Natl Acad Sci USA 91:10380-10383.

Allen K, Rawlins JNP, Bannerman DM, Csicsvari J (2012) Hippocampal place cells can encode multiple trial-dependent features through rate remapping. J Neurosci 32:14752-14766.

Aoki Y, Igata H, Ikegaya Y, Sasaki T (2019) The integration of goal-directed signals onto spatial maps of hippocampal place cells. Cell Rep 27:1516-1527.

Atluri PP, Regehr WG (1996) Determinants of the time course of facilitation at the granule cell to purkinje cell synapse. J Neurosci 16:5661-5671.
Attwell D, Laughlin SB (2001) An energy budget for signaling in the grey matter of the brain. J Cereb Blood Flow Metab 21:11331145.

Atwood HL, Karunanithi S (2002) Diversification of synaptic strength: presynaptic elements. Nat Rev Neurosci 3:497-516.

Bacaj T, Wu D, Yang X, Morishita W, Zhou P, Xu W, Malenka RC, Südhof TC (2013) Synaptotagmin-1 and synaptotagmin-7 trigger synchronous and asynchronous phases of neurotransmitter release. Neuron 80:947-959.

Balasubramanian V, Kimber D, Berry MJ (2001) Metabolically efficient information processing. Neural Comput 13:799-815.

Basu J, Siegelbaum SA (2015) The corticohippocampal circuit, synaptic plasticity, and memory. Cold Spring Harb Perspect Biol 7: a021733.

Bliss TVP, Collingridge GL (2013) Expression of NMDA receptor-dependent LTP in the hippocampus: bridging the divide. Mol Brain 6:5.

Borst JGG (2010) The low synaptic release probability in vivo. Trends Neurosci 33:259-266.

Branco T, Staras K, Darcy KJ, Goda Y (2008) Local dendritic activity sets release probability at hippocampal synapses. Neuron 59:475485.

Cai Y, Gavornik JP, Cooper LN, Yeung LC, Shouval HZ (2007) Effect of stochastic synaptic and dendritic dynamics on synaptic plasticity in visual cortex and hippocampus. J Neurophysiol 97:375-386.

Chklovskii DB, Schikorski T, Stevens CF (2002) Wiring optimization in cortical circuits. Neuron 34:341-347.

Choi JH, Sim SE, Kim JI, Choi DI, Oh J, Ye S, Lee J, Kim T, Ko HG, Lim CS, Kaang BK (2018) Interregional synaptic maps among engram cells underlie memory formation. Science 360:430-435.

Costa RP, Padamsey Z, D'Amour JA, Emptage NJ, Froemke RC, Vogels TP (2017) Synaptic transmission optimization predicts expression loci of long-term plasticity. Neuron 96:177-189.

Cover TM, Thomas JA (2012) Elements of information theory. Hoboken: Wiley.

Davis GW, Müller M (2015) Homeostatic control of presynaptic neurotransmitter release. Annu Rev Physiol 77:251-270.

Debanne D, Guerineau NC, Gahwiler BH, Thompson SM (1995) Physiology and pharmacology of unitary synaptic connections between pairs of cells in areas ca3 and ca1 of rat hippocampal slice cultures. J Neurophysiol 73:1282-1294.

Deco G, Rolls ET, Romo R (2009) Stochastic dynamics as a principle of brain function. Prog Neurobiol 88:1-16.

Dittman JS, Kreitzer AC, Regehr WG (2000) Interplay between facilitation, depression, and residual calcium at three presynaptic terminals. J Neurosci 20:1374-1385.

Dobrunz LE, Stevens CF (1997) Heterogeneity of release probability, facilitation, and depletion at central synapses. Neuron 18:9951008.

Dobrunz LE, Stevens CF (1999) Response of hippocampal synapses to natural stimulation patterns. Neuron 22:157-166.

Emes RD, Grant SGN (2012) Evolution of synapse complexity and diversity. Annu Rev Neurosci 35:111-131.

Emptage NJ, Reid CA, Fine A (2001) Calcium stores in hippocampal synaptic boutons mediate short-term plasticity, store-operated ca2 + entry, and spontaneous transmitter release. Neuron 29:197208.

Enoki R, Hu YL, Hamilton D, Fine A (2009) Expression of long-term plasticity at individual synapses in hippocampus is graded, bidirectional, and mainly presynaptic: optical quantal analysis. Neuron 62:242-253.

Fenton AA, Muller RU (1998) Place cell discharge is extremely variable during individual passes of the rat through the firing field. Proc Natl Acad Sci USA 95:3182-3187.

Fenton AA, Lytton WW, Barry JM, Lenck-Santini PP, Zinyuk LE, Kubík S, Bures J, Poucet B, Muller RU, Olypher AV (2010) Attention-like modulation of hippocampus place cell discharge. $\mathrm{J}$ Neurosci 30:4613-4625.

Fernández-Chacón R, Königstorfer A, Gerber SH, García J, Matos MF, Stevens CF, Brose N, Rizo J, Rosenmund C, Südhof TC 
(2001) Synaptotagmin I functions as a calcium regulator of release probability. Nature 410:41-49.

Fernández de Sevilla D, Buño W (2003) Presynaptic inhibition of schaffer collateral synapses by stimulation of hippocampal cholinergic afferent fibres. Eur J Neurosci 17:555-558.

Geppert M, Goda Y, Hammer RE, Li C, Rosahl TW, Stevens CF, Südhof TC (1994) Synaptotagmin I: a major ca2+ sensor for transmitter release at a central synapse. Cell 79:717-727.

Goldman MS (2004) Enhancement of information transmission efficiency by synaptic failures. Neural Comput 16:1137-1162.

Grieves RM, Wood ER, Dudchenko PA (2016) Place cells on a maze encode routes rather than destinations. Elife 5:e15986.

Gruart A, Muñoz MD, Delgado-García JM (2006) Involvement of the ca3-ca1 synapse in the acquisition of associative learning in behaving mice. J Neurosci 26:1077-1087.

Hamid E, Church E, Alford S (2019) Quantitation and simulation of single action potential-evoked ca2 + signals in ca1 pyramidal neuron presynaptic terminals. eNeuro 6:ENEURO.0343-19.2019.

Harris JJ, Jolivet R, Attwell D (2012) Synaptic energy use and supply. Neuron 75:762-777.

Harris JJ, Jolivet R, Engl E, Attwell D (2015) Energy-efficient information transfer by visual pathway synapses. Curr Biol 25:3151-3160.

Harris JJ, Engl E, Attwell D, Jolivet RB (2019) Energy-efficient information transfer at thalamocortical synapses. PLoS Comput Biol 15:e1007226.

Hasenstaub A, Otte S, Callaway E, Sejnowski TJ (2010) Metabolic cost as a unifying principle governing neuronal biophysics. Proc Natl Acad Sci USA 107:12329-12334.

Hennig MH (2013) Theoretical models of synaptic short term plasticity. Front Comput Neurosci 7:45.

Henze DA, Wittner L, Buzsáki G (2002) Single granule cells reliably discharge targets in the hippocampal ca3 network in vivo. Nat Neurosci 5:790-795.

Holderith N, Lorincz A, Katona G, Rózsa B, Kulik A, Watanabe M, Nusser Z (2012) Release probability of hippocampal glutamatergic terminals scales with the size of the active zone. Nat Neurosci 15:988-997.

Jackman SL, Turecek J, Belinsky JE, Regehr WG (2016) The calcium sensor synaptotagmin 7 is required for synaptic facilitation. Nature 529:88-91.

James B, Darnet L, Moya-Díaz J, Seibel S-H, Lagnado L (2019) An amplitude code transmits information at a visual synapse. Nat Neurosci 22:1140-1147.

Kandaswamy U, Deng P-Y, Stevens CF, Klyachko VA (2010) The role of presynaptic dynamics in processing of natural spike trains in hippocampal synapses. J Neurosci 30:15904-15914.

Klyachko VA, Stevens CF (2006) Excitatory and feed-forward inhibitory hippocampal synapses work synergistically as an adaptive filter of natural spike trains. PLoS Biol 4:e207.

Laughlin SB (2001) Energy as a constraint on the coding and processing of sensory information. Curr Opin Neurobiol 11:475-480.

Laughlin SB, Sejnowski TJ (2003) Communication in neuronal networks. Science 301:1870-1874.

Laughlin SB, de Ruyter van Steveninck RR, Anderson JC (1998) The metabolic cost of neural information. Nat Neurosci 1:36-41.

Letellier M, Park YK, Chater TE, Chipman PH, Gautam SG, OshimaTakago T, Goda Y (2016) Astrocytes regulate heterogeneity of presynaptic strengths in hippocampal networks. Proc Natl Acad Sci USA 113:E2685-E2694.

Leutgeb S, Leutgeb JK, Barnes CA, Moser El, McNaughton BL, Moser M-B (2005) Independent codes for spatial and episodic memory in hippocampal neuronal ensembles. Science 309:619623.

Levina A, Herrmann JM, Geisel T (2007) Dynamical synapses causing self-organized criticality in neural networks. Nature Phys 3:857860.

Levy WB, Baxter RA (2002) Energy-efficient neuronal computation via quantal synaptic failures. J Neurosci 22:4746-4755.

Lisman JE (1997) Bursts as a unit of neural information: making unreliable synapses reliable. Trends Neurosci 20:38-43.
Malenka RC, Bear MF (2004) Ltp and Itd: an embarrassment of riches. Neuron 44:5-21.

Manwani A, Koch C (2001) Detecting and estimating signals over noisy and unreliable synapses: information-theoretic analysis. Neural Comput 13:1-33.

Matveev V, Wang XJ (2000) Differential short-term synaptic plasticity and transmission of complex spike trains: to depress or to facilitate? Cereb Cortex 10:1143-1153.

McNaughton BL, Morris RGM (1987) Hippocampal synaptic enhancement and information storage within a distributed memory system. Trends Neurosci 10:408-415.

Mejias JF, Torres JJ (2009) Maximum memory capacity on neural networks with short-term synaptic depression and facilitation. Neural Comput 21:851-871.

Mizuseki K, Royer S, Diba K, Buzsáki G (2012) Activity dynamics and behavioral correlates of ca3 and ca1 hippocampal pyramidal neurons. Hippocampus 22:1659-1680.

Mongillo G, Barak O, Tsodyks M (2008) Synaptic theory of working memory. Science 319:1543-1546.

Moser El, Krobert KA, Moser MB, Morris RGM (1998) Impaired spatial learning after saturation of long-term potentiation. Science 281:2038-2042.

Murthy VN, Sejnowski TJ, Stevens CF (1997) Heterogeneous release properties of visualized individual hippocampal synapses. Neuron 18:599-612.

Nadkarni S, Bartol TM, Sejnowski TJ, Levine H (2010) Modelling vesicular release at hippocampal synapses. PLoS Comput Biol 6: e1000983.

Nadkarni S, Bartol TM, Stevens CF, Sejnowski TJ, Levine H (2012) Short-term plasticity constrains spatial organization of a hippocampal presynaptic terminal. Proc Natl Acad Sci USA 109:1465714662.

Newman CM (1988) Memory capacity in neural network models: rigorous lower bounds. Neural Netw 1:223-238.

O'Keefe J, Dostrovsky J (1971) The hippocampus as a spatial map. Preliminary evidence from unit activity in the freely-moving rat. Brain Res 34:171-175.

Olypher AV, Lánský P, Fenton AA (2002) Properties of the extra-positional signal in hippocampal place cell discharge derived from the overdispersion in location-specific firing. Neuroscience 111:553566.

Pfister JP, Dayan P, Lengyel M (2010) Synapses with short-term plasticity are optimal estimators of presynaptic membrane potentials. Nat Neurosci 13:1271-1275.

Qian J, Saggau P (1997) Presynaptic inhibition of synaptic transmission in the rat hippocampus by activation of muscarinic receptors: involvement of presynaptic calcium influx. $\mathrm{Br} J$ Pharmacol 122:511-519.

Rosenbaum R, Rubin J, Doiron B (2012) Short term synaptic depression imposes a frequency dependent filter on synaptic information transfer. PLoS Comput Biol 8:e1002557.

Rotman Z, Deng PY, Klyachko VA (2011) Short-term plasticity optimizes synaptic information transmission. J Neurosci 31:1480014809.

Salmasi M, Loebel A, Glasauer S, Stemmler M (2019a) Short-term synaptic depression can increase the rate of information transfer at a release site. PLoS Comput Biol 15:e1006666.

Salmasi M, Stemmler M, Glasauer S, Loebel A (2019b) Synaptic information transmission in a two-state model of short-term facilitation. Entropy 21:756.

Schikorski T, Stevens CF (1997) Quantitative ultrastructural analysis of hippocampal excitatory synapses. J Neurosci 17:5858-5867.

Shapiro ML, Tanila H, Eichenbaum H (1997) Cues that hippocampal place cells encode: dynamic and hierarchical representation of local and distal stimuli. Hippocampus 7:624-642.

Soares C, Lee KFH, Béïque J-C (2017) Metaplasticity at ca1 synapses by homeostatic control of presynaptic release dynamics. Cell Rep 21:1293-1303.

Stevens CF, Wang Y (1994) Changes in reliability of synaptic function as a mechanism for plasticity. Nature 371:704-707. 
Stevens CF, Wang Y (1995) Facilitation and depression at single central synapses. Neuron 14:795-802.

Thomson AM (2000) Facilitation, augmentation and potentiation at central synapses. Trends Neurosci 23:305-312.

Tkačik G, Bialek W (2016) Information processing in living systems. Annu Rev Condens Matter Phys 7:89-117.

Tsodyks MV, Markram H (1997) The neural code between neocortical pyramidal neurons depends on neurotransmitter release probability. Proc Natl Acad Sci USA 94:719-723.

Varshney LR, Sjöström PJ, Chklovskii DB (2006) Optimal information storage in noisy synapses under resource constraints. Neuron 52:409-423.

Vincent BT, Baddeley RJ (2003) Synaptic energy efficiency in retinal processing. Vision Res 43:1285-1292.
Vyleta NP, Jonas P (2014) Loose coupling between ca2+ channels and release sensors at a plastic hippocampal synapse. Science 343:665-670.

Zhang C, Peskin CS (2015) Improved signaling as a result of randomness in synaptic vesicle release. Proc Natl Acad Sci USA 112:14954-14959.

Zhang C, Wu B, Beglopoulos V, Wines-Samuelson M, Zhang D, Dragatsis I, Südhof TC, Shen J (2009) Presenilins are essential for regulating neurotransmitter release. Nature 460:632-636.

Zhao C, Dreosti E, Lagnado L (2011) Homeostatic synaptic plasticity through changes in presynaptic calcium influx. J Neurosci 31:7492-7496.

Zucker RS, Regehr WG (2002) Short-term synaptic plasticity. Annu Rev Physiol 64:355-405. 